\title{
Abordaje del tabaquismo en centros de tratamiento de drogodependencias: implicaciones clínicas y recomendaciones para su implementación
}

\section{Smoking cessation interventions in substance use treatment facilities: clinical implications and recommendations for implementation}

\author{
Alba González-Roz*; lucía Ruano*; Gema Aonso-Diego*; Ángel García-Pérez*; \\ SARA WeidberG*; Roberto Secades-Villa*. \\ *Departamento de Psicología. Universidad de Oviedo (España).
}

$\mathrm{E}$ 1 impacto positivo de la cesación tabáquica en los resultados de abstinencia de uso de sustancias acumula una evidencia creciente. Dejar de fumar se relaciona con un $25 \%$ más de probabilidad de mantener la abstinencia a largo plazo. Por el contrario, fumar se asocia con una probabilidad 4,86 veces mayor de recaída en el consumo de otras sustancias (Weinberger et al., 2018). A pesar del consenso a favor de la implementación de servicios de cesación tabáquica en centros de tratamiento de drogodependencias (Cohn, Elmasry, y Niaura, 2017), la implementación del tratamiento del tabaquismo ha tenido una adopción escasa en estos contextos.En España, se considera que la cultura organizacional de los centros de drogodependencias supone la principal barrera para la integración de los servicios de cesación tabáquica. La implementación del tratamiento del tabaquismo ha tenido una adopción escasa en estos contextos. La percepción de que el tabaco es una sustancia menos nociva que el alcohol. La creencia de que la cesación tabáquica comprometerá la recuperación del abuso de sustancias desanima a los pacientes a dejar de fumar.

Este estudio tuvo como objetivo avanzar en el conocimiento sobre la oferta de tratamientos para el abordaje del tabaquismo en centros de tratamiento de uso de sustancias (TUS) en España. También como objetivo secundario se identificaron las barreras para implementación de tratamientos de cesación tabáquica.
Identificamos un total de 20 centros de tratamiento de TUS en el Principado de Asturias (ubicados en el noroeste de España, con 1.034 millones de habitantes) a través de una guía de recursos en drogodependencias (Consejería de Sanidad del Gobierno del Principado de Asturias, 2016). De los 20 centros existentes, 15 acordaron participar (7 de 12 ambulatorios, 5 de 5 residenciales y 3 de 3 centros mixtos). El tipo de tratamiento principal era mixto (14/15), seguido de centros para mujeres $(1 / 15)$. La mayoría de los programas de tratamiento se centraban en tres o más sustancias $(10 / 15)$, seguido de solo cocaína $(2 / 15)$, cocaína y opiáceos $(1 / 15)$, solo cannabis $(1 / 15)$ y solo alcohol $(1 / 15)$. Se elaboró una encuesta ad-hoc que fue proporcionada al coordinador principal de cada centro entre julio y octubre de 2018 (íntegramente accesible en https://docs. google.com/forms/d/1y1MtPzmQQptSHVHOpRsd2dBOaAGmf9NzRJw5Ih9UFkA/edit?ts=5c86aee2), compuesta de 34 ítems, para recopilar datos sobre la disponibili$\mathrm{dad} /$ oferta de recursos para apoyar a los pacientes en su intento de dejar de fumar y sobre la normativa relacionada con el consumo de tabaco. También se pidió a los coordinadores su opinión respecto a la oferta sistemática de tratamientos para dejar de fumar a los pacientes con TUS.

De los 15 centros encuestados, solo 3 (20\%) ofrecían cesación tabáquica de manera sistemática. De los 15 centros, $10(66,66 \%)$ autoinformaron de alguna medida de control de tabaco: prohibición del intercambio de cigarrillos (4/10; 
$40 \%)$, limitación del tiempo permitido para liar cigarrillos $(4 / 10 ; 40 \%)$, y uso de cigarrillos como premio/castigo $(2 / 10 ; 20 \%)$. La Tabla 1 muestra las barreras que limitan la oferta sistemática de tratamientos para dejar de fumar.

Tabla 1. Barreras a la implementación efectiva de tratamientos de cesación tabáquica $(N=15)$

\begin{tabular}{|c|c|c|}
\hline \multirow[t]{2}{*}{$\begin{array}{l}\text { Nivel del } \\
\text { personal (n/\%) }\end{array}$} & $\begin{array}{l}\text { Creencias negativas respecto al efecto } \\
\text { de dejar de fumar sobre la abstinencia } \\
\text { del uso de sustancias }\end{array}$ & $11(73,3 \%)$ \\
\hline & Falta de formación específica & $2(13,3 \%)$ \\
\hline $\begin{array}{l}\text { Nivel de recursos } \\
(\mathrm{n} / \%)\end{array}$ & $\begin{array}{l}\text { Falta de recursos económicos y } \\
\text { temporales }\end{array}$ & $3(20 \%)$ \\
\hline \multirow[t]{2}{*}{$\begin{array}{l}\text { Nivel de } \\
\text { organización } \\
\text { (n/\%) }\end{array}$} & $\begin{array}{l}\text { Falta de consideración de la } \\
\text { consecución de la abstinencia como } \\
\text { parte del plan terapéutico }\end{array}$ & $9(60 \%)$ \\
\hline & $\begin{array}{l}\text { Ausencia de restricciones en el } \\
\text { número de CPD }\end{array}$ & $7(46,6 \%)$ \\
\hline
\end{tabular}

Nota TUS = Trastorno por uso de sustancias; $\mathrm{CPD}=$ Cigarillos por día.

La escasa oferta sistemática para el tratamiento del tabaquismo ofertada por los centros de tratamiento de drogodependencias es similar que indicó que el 55,6\% de los 142 centros de tratamiento de drogodependencias españoles ofrecían apoyo para la cesación tabáquica (Becoña Iglesias et al., 2006). Demorar la oferta del tratamiento del tabaquismo en los contextos de tratamiento de drogodependencias se traduce en la omisión de una ayuda que hubiera resultado de interés para muchos pacientes.

Identificamos varios factores que reducen la probabilidad de abordar el tabaquismo de los pacientes: creencias negativas respecto al impacto que tiene dejar de fumar sobre la abstinencia de otras sustancias, falta de políticas de espacios libres de humo, inexistencia de formación para el personal, y una cultura permisiva en cuanto al consumo de tabaco.

El desarrollo y la evaluación de intervenciones centradas en el cambio organizacional de los centros de tratamiento han mostrado ser coste-efectivas para favorecer un incremento en la oferta sistemática del tratamiento del tabaquismo a los pacientes (Skelton et al., 2018). Implementar cambios organizacionales con el fin de "desnormalizar" el uso de tabaco, ofrecer entrenamiento específico sobre el abordaje del tabaquismo al personal y eliminar las creencias negativas respecto al efecto que tiene dejar de fumar en la abstinencia de otras sustancias son todas medidas altamente recomendables.

Las siguientes limitaciones deben ser mencionadas. El hecho que no recopilamos datos de los pacientes y el personal podría haber tenido un impacto negativo en la representatividad de cada centro. Además, debido a que el estudio se restringió a Asturias los datos obtenidos no se pueden generalizar a otras comunidades. Por último, los centros de hospitalización fueron excluidos, y los resultados no pueden extrapolarse a estos contextos.
En resumen, estos hallazgos representan una contribución importante a la situación actual en materia de tratamiento del tabaquismo en los centros de drogodependencias. Con el fin de integrar el tratamiento del tabaquismo en los centros de drogodependencias se han de realizar intervenciones que impacten sobre las variables a nivel de paciente, del personal trabajador y la organización.

\section{Reconocimientos}

Mostramos nuestro profundo agradecimiento a los coordinadores que participaron en este estudio.

\section{Conflicto de intereses}

Los autores declaran la inexistencia de conflictos de intereses.

\section{Fuentes de financiación}

Este estudio recibió apoyo del Plan Nacional sobre Drogas (PNSD: Ref. MSSSI-17-2013I036) y del Ministerio de Ciencia, Investigación y Universidades (BES-2016-076663; FPU17/00659; FPU15/04327).

Las entidades financiadoras no asumieron papel alguno en el diseño del estudio o en la recolección, análisis e interpretación de los datos, en la redacción del manuscrito, o en cualquier decisión relacionada con la presentación del manuscrito para su publicación.

\section{Referencias}

Becoña Iglesias, E., García García, V., de Echave Sanz, J., Fernández Miranda, J., Sánchez Fernández, M., Terán Prieto, A., . . Castillo, A. (2006). Tratamiento del tabaquismo en los centros de drogodependencias y alcoholismo en España. Estado de la situación y perspectivas futuras. Adicciones, 18, 23-28. doi:10.20882/adicciones.353.

Cohn, A., Elmasry, H. y Niaura, R. (2017). Facility-level, state, and financial factors associated with changes in the provision of smoking cessation services in US substance abuse treatment facilities: Results from the national survey of substance abuse treatment rervices 2006 to 2012. Journal of Substance Abuse Treatment, 77, 107-114. doi:10.1016/j.jsat.2017.03.014.

Consejería de Sanidad del Gobierno del Principado de Asturias. (2016). Guía de recursos en drogodependencias del Principado de Asturias (cuarta edición). [Guide to resources on drug addiction of the Health Council of the Principality of Asturias, fourth edition]. Recuperado de https://www.astursalud.es/documents/31867/36150/ $\mathrm{Gu} \% \mathrm{C} 3 \% \mathrm{ADa}+\mathrm{de}+$ recursos+en+drogodependencias. pdf/efa9e9d3-5013-0cf1-0ccb-bb8a64bd04ab. 
Nieva, G. y Gual, A. (2005). El tratamiento del tabaquismo en alcohólicos: ¿miopía o negligencia? Adicciones, 17, 177-180. doi:10.20882/adicciones.366

Prochaska, J. J., Delucchi, K. y Hall, S. M. (2004). A meta-analysis of smoking cessation interventions with individuals in substance abuse treatment or recovery. Journal of Consulting and Clinical Psychology, 72, 1144-1156. doi:10.1037/0022-006X.72.6.1144.

Skelton, E., Tzelepis, F., Shakeshaft, A., Guillaumier, A., McCrabb, S. y Bonevski, B. (2018). Integrating smoking cessation care in alcohol and other drug treatment settings using an organizational change intervention: a systematic review. Addiction, 113, 2158-2172. doi:10.1111/ add.14369.

Weinberger, A. H., Gbedemah, M., Wall, M. M., Hasin, D. S., Zvolensky, M. J. y Goodwin, R. D. (2018). Cigarette use is increasing among people with illicit substance use disorders in the United States, 2002-14: emerging disparities in vulnerable populations. Addiction, 113, 719-728. doi:10.1111/add.14082. 